PROCEEDINGS OF THE

AMERICAN MATHEMATICAL SOCIETY

Volume 135, Number 12, December 2007, Pages 3833-3836

S 0002-9939(07)08907-1

Article electronically published on August 17, 2007

\title{
ON POSITIVE UNIPOTENT OPERATORS ON BANACH LATTICES
}

\author{
ROMAN DRNOVŠEK
}

(Communicated by Joseph A. Ball)

\begin{abstract}
Let $T$ be a positive operator on a complex Banach lattice. We prove that $T$ is greater than or equal to the identity operator $I$ if$$
\lim _{n \rightarrow \infty} n\left\|(T-I)^{n}\right\|^{1 / n}=0 .
$$

\section{INTRODUCTION}

Throughout the paper, $E$ denotes a complex Dedekind complete Banach lattice, i.e., $E=\operatorname{Re} E \oplus \operatorname{Re} E$, where $\operatorname{Re} E$ is a real Dedekind complete Banach lattice. Denote by $\mathcal{L}_{r}(E)$ the space of all regular operators on $E$, i.e., those operators that can be expressed as linear combinations of positive operators. This space is a subspace of the space $\mathcal{L}(E)$ of all bounded linear operators on $E$, and it is also a complex Banach lattice algebra with respect to the regular norm that is defined by $\|T\|_{r}:=\||T|\|$. The center $\mathcal{Z}(E)$ defined by $\mathcal{Z}(E):=\left\{T \in \mathcal{L}_{r}(E)\right.$ : $|T| \leq \lambda I$ for some $\lambda \geq 0\}$ is a commutative full subalgebra of the space $\mathcal{L}(E)$. If $T \in \mathcal{Z}(E)$, then $\|T\|=\|T\|_{r}=\min \{\lambda \geq 0:|T| \leq \lambda I\}$. Since $\mathcal{Z}(E)$ is also a band in $\mathcal{L}_{r}(E)$, we have the band decomposition $\mathcal{L}_{r}(E)=\mathcal{Z}(E) \oplus \mathcal{Z}(E)^{d}$. The associated band projection in $\mathcal{L}_{r}(E)$ onto $\mathcal{Z}(E)$ is denoted by $\mathcal{D}$, and it is called the diagonal map. By a result of Voigt $[8, \mathcal{D}$ is a contraction with respect to the operator norm, i.e., $\|\mathcal{D}(T)\| \leq\|T\|$ for all $T \in \mathcal{L}_{r}(E)$. It is not difficult to verify that $0 \leq \mathcal{D}(T) \leq r(T) I$ for any positive operator $T$ on $E$, where $r(T)$ denotes the spectral radius of $T$. Other interesting properties of the diagonal map are explored in the paper [3. For the terminology not explained in the text we refer e.g. to the books 9, 1, and 4.

An operator is said to be unipotent whenever its spectrum contains only the number 1 . In this note we give a partial answer to the question posed by Huijsmans and de Pagter: Is it true that $T \geq I$ for every unipotent positive operator $T$ on $E$ ? This question was studied in the papers [11, [10] and [5]. In particular, Zhang [10] showed that the answer is affirmative under the additional hypothesis that there exist $\alpha \in\left(0, \frac{1}{2}\right)$ and a constant $c \geq 0$ such that $\left\|T^{-n}\right\|=O\left(\exp \left(c n^{\alpha}\right)\right)$ as $n \rightarrow \infty$. In this paper we use the same method to prove a similar result replacing

Received by the editors December 1, 2005 and, in revised form, August 23, 2006.

2000 Mathematics Subject Classification. Primary 47B65, 47A10.

Key words and phrases. Banach lattices, positive operators, spectrum.

This work was supported in part by the Ministry of Higher Education, Science and Technology of Slovenia.

(C)2007 American Mathematical Society Reverts to public domain 28 years from publication 
the assumption on the growth of the negative powers of $T$ by a nice assumption on the growth of the powers of the quasinilpotent operator $A=T-I$.

\section{REsults}

The main result of the paper is the following.

Theorem 1. Let $T$ be a positive operator on $E$, and let $A=T-I$. If

$$
\lim _{n \rightarrow \infty} n\left\|\mathcal{D}\left(A^{n}\right)\right\|^{1 / n}=0
$$

and if $\mathcal{D}\left(T^{n}\right) \leq I$ for all $n \in \mathbb{N}$, then the operator $A$ is positive.

Proof. Let us consider the operator valued function $f$ defined by

$$
f(z)=\mathcal{D}\left(e^{z A}\right) .
$$

Since the diagonal map $\mathcal{D}$ is continuous with respect to the operator norm, $f$ is an entire function, and we have

$$
f(z)=\mathcal{D}\left(\sum_{n=0}^{\infty} \frac{z^{n} A^{n}}{n !}\right)=\sum_{n=0}^{\infty} c_{n} z^{n},
$$

where

$$
c_{n}=\frac{\mathcal{D}\left(A^{n}\right)}{n !} \quad(n=0,1,2,3, \ldots) .
$$

We claim that $f$ is an entire function of minimal type with respect to the order $1 / 2$. Choose $\varepsilon>0$. Then $\left\|\mathcal{D}\left(A^{n}\right)\right\| \leq\left(\frac{\varepsilon}{n}\right)^{n}$ for large $n \in \mathbb{N}$. Since $n ! \geq\left(\frac{n}{e}\right)^{n}$ for all $n \in \mathbb{N}$, we have

$$
\left\|c_{n}\right\|=\frac{\left\|\mathcal{D}\left(A^{n}\right)\right\|}{n !} \leq \frac{\varepsilon^{n} e^{n}}{n^{2 n}}
$$

for large $n \in \mathbb{N}$. Since the order $\rho$ of $f$ is determined by the formula (see [2])

we have

$$
\rho=\limsup _{n \rightarrow \infty} \frac{n \log n}{\log \left(1 /\left\|c_{n}\right\|\right)}
$$

$$
\rho \leq \limsup _{n \rightarrow \infty} \frac{n \log n}{2 n \log n-n \log \varepsilon-n}=\frac{1}{2} .
$$

If $\rho$ equals $1 / 2$, then the following estimate for the type $\sigma$ of $f$ holds:

$$
\sigma=\frac{1}{\rho e} \limsup _{n \rightarrow \infty}\left(n\left\|c_{n}\right\|^{\rho / n}\right) \leq \frac{2 \sqrt{\varepsilon}}{\sqrt{e}}
$$

which implies that $\sigma=0$. This proves our claim.

Now, for any nonnegative number $x$ we have

$$
0 \leq f(x)=e^{-x} \mathcal{D}\left(e^{x T}\right)=e^{-x} \sum_{n=0}^{\infty} \mathcal{D}\left(T^{n}\right) \frac{x^{n}}{n !} \leq e^{-x} \sum_{n=0}^{\infty} \frac{x^{n}}{n !} I=I .
$$

It follows that the operator valued entire function $g$ defined by $g(z)=f\left(z^{2}\right)$ is of minimal type with respect to the order 1 and is bounded on the real axis. By the Phragmén-Lindelöf theorem (see [2, remark 3 on p. 39]), we conclude that $g$ and $f$ are constant functions. Since $f(0)=I$, we obtain that $f(x)=I$ for any nonnegative number $x$. This implies that $\mathcal{D}\left(T^{n}\right)=I$ for each $n$, so that $\mathcal{D}(A)=0$, completing the proof of the theorem. 
We now state and prove three corollaries. The first of them provides a partial answer to the question mentioned above.

Corollary 1. Let $T$ be a positive operator on $E$, and let $A=T-I$. If

$$
\lim _{n \rightarrow \infty} n\left\|A^{n}\right\|^{1 / n}=0,
$$

then $A$ is positive.

Proof. Since $A$ is quasinilpotent, $T$ and its powers are unipotent operators. It follows that $\mathcal{D}\left(T^{n}\right) \leq r\left(T^{n}\right) I=I$ for all $n \in \mathbb{N}$. Since the assumption implies that $\lim _{n \rightarrow \infty} n\left\|\mathcal{D}\left(A^{n}\right)\right\|^{1 / n}=0$, we can apply Theorem 1 to complete the proof.

Corollary 2. Let $T$ be a positive operator on $E$ that is power bounded, i.e., $\sup \left\{\left\|T^{n}\right\|: n \in \mathbb{N}\right\}<\infty$. If the operator $A=T-I$ satisfies the condition $\lim _{n \rightarrow \infty} n\left\|\mathcal{D}\left(A^{n}\right)\right\|^{1 / n}=0$ (in particular, $\lim _{n \rightarrow \infty} n\left\|A^{n}\right\|^{1 / n}=0$ ), then $T=I$.

Proof. If we denote $c=\sup \left\{\left\|T^{n}\right\|: n \in \mathbb{N}\right\}$, then $\left\|\mathcal{D}\left(T^{n}\right)\right\| \leq\left\|T^{n}\right\| \leq c$ for all $n \in \mathbb{N}$, so that $\mathcal{D}\left(T^{n}\right) \leq c I$ for all $n \in \mathbb{N}$. Since $\mathcal{D}\left(T^{n}\right)^{m} \leq \mathcal{D}\left(T^{n m}\right) \leq c I$ for all $m, n \in \mathbb{N}$, we conclude that $\mathcal{D}\left(T^{n}\right) \leq I$ for all $n \in \mathbb{N}$. Now the operator $A$ is positive by Theorem 1. Since $T^{n}=(I+A)^{n} \geq n A$ for all $n \in \mathbb{N}$, we have $n\|A\| \leq\left\|T^{n}\right\|$ for all $n \in \mathbb{N}$, so that $A=0$.

Corollary 3. Let $T$ be a positive operator on a Banach lattice $E$ that is of the form $\lambda I+K$, where $\lambda \geq 0$ and $K$ is a compact operator. If $\lim _{n \rightarrow \infty} n\left\|K^{n}\right\|^{1 / n}=0$, then $T$ has a non-trivial invariant closed ideal.

Proof. An application of Theorem 1 gives that the operator $K$ is positive. Since $K$ is a quasinilpotent operator, $K$ (and $T$ as well) has a non-trivial invariant closed ideal by the well-known result of de Pagter (see [6] or [4, theorem 4.2.2]).

We remark that the compactness assumption in the last result cannot be omitted. Indeed, Schaefer (see [7] or [4, section 4.2]) constructed examples of quasinilpotent positive operators with no non-trivial invariant closed ideals. It is easy to see that among them there is an operator $T$ satisfying the condition $\lim _{n \rightarrow \infty} n\left\|T^{n}\right\|^{1 / n}=0$.

\section{REFERENCES}

[1] C.D. Aliprantis, O. Burkinshaw, Positive operators, Academic Press, Orlando 1985. MR $809372(87 \mathrm{~h}: 47086)$

[2] B. Ya. Levin, Lectures on entire functions, Translations of Mathematical Monographs 150, American Mathematical Society, Providence, 1996. MR.1400006 (97j:30001)

[3] W. A. J. Luxemburg, B. de Pagter, A. R. Schep, Diagonals of the powers of an operator on a Banach lattice, in: Operator theory in function spaces and Banach lattices, 223-273, Oper. Theory Adv. Appl. 75, Birkhäuser, Basel, 1995. MR1322506 (97i:47076)

[4] P. Meyer-Nieberg, Banach lattices, Springer-Verlag, Berlin, 1991. MR.1128093 (93f:46025)

[5] S. Mouton, A spectral problem in ordered Banach algebras, Bull. Austral. Math. Soc. 67 (2003), no. 1, 131-144. MR1962967 (2004d:47075)

[6] B. de Pagter, Irreducible compact operators, Math. Z. 192 (1986), 149-153. MR835399 (87d:47052)

[7] H.H. Schaefer, Topologische Nilpotenz irreduzibler Operatoren, Math. Z. 117 (1970), 135140. MR0276802 (43:2542)

[8] J. Voigt, The projection onto the center of operators in a Banach lattice, Math. Z. 199 (1988), no. 1, 115-117. MR.954756 (89f:47058)

[9] A.C. Zaanen, Riesz spaces II, North Holland, Amsterdam, 1983. MR704021 (86b:46001)

[10] X. D. Zhang, On spectral properties of positive operators, Indag. Math. (N.S.) 4 (1993), no. 1, 111-127. MR.1213328 (94b:47047) 
[11] X. D. Zhang, Some aspects of the spectral theory of positive operators, Positive operators and semigroups on Banach lattices (Curaçao, 1990). Acta Appl. Math. 27 (1992), no. 1-2, 135-142. MR1184885 (93j:47056)

Department of Mathematics, University of Ljubljana, Jadranska 19. Si-1000 LjublJANA, Slovenia

E-mail address: Roman.Drnovsek@fmf.uni-lj.si 